\title{
The Effect of Laser Wavelength on Sensitivity and Accuracy of the Evanescent-Based Blood Glucose Sensor \\ Nola Fridayanti and Muldarisnur
}

Physics Department, faculty of mathematics and Natural Sciences, Andalas University, Padang, West Sumatera, 25163, Indonesia

\section{Abstract}

We have developed bent fiber optic based sensors for non-invasive glucose level measurement. The developed sensor employs the attenuation of evanescent wave in the optical fiber due to its interaction with glucose molecules in urine samples. The attenuation is proportional to the concentration of the glucose molecules. We investigated dependence of the sensitivity and the accuracy of the sensor on the wavelength of diode laser source. Three diode lasers (i.e. red laser $=650 \mathrm{~nm}$, green laser $=532 \mathrm{~nm}$, and violet laser $=400 \mathrm{~nm}$ ) were used for measurements. The attenuated

Corresponding Author:

Muldarisnur

muldarisnur@sci.unand.ac.id

Received: 19 February 2019

Accepted: 5 March 2019

Published: 16 April 2019

Publishing services provided by

Knowledge $\mathrm{E}$

(c) Nola Fridayanti and

Muldarisnur. This article is

distributed under the terms of

the Creative Commons

Attribution License, which

permits unrestricted use and

redistribution provided that the

original author and source are

credited.

Selection and Peer-review under the responsibility of the ICBSA Conference Committee. light in the fiber optic was detected by a photodiode and then converted to electrical signal. The interaction of evanescent wave interaction with glucose molecules was enhanced by partial removing of fiber optic cladding. The length of cladding removal was varied between $1 \mathrm{~cm}$ and $4 \mathrm{~cm}$ while bending radius was kept constant at $3.5 \mathrm{~cm}$. We found that the diode laser wavelength influences the sensitivity and the accuracy of blood glucose sensor significantly. The highest sensitivity of $0.9 \mathrm{mV} /(\mathrm{mg} / \mathrm{dL})$ is obtained for sensor using red laser with the length of cladding removal of $2 \mathrm{~cm}$. The sensitivity of the sensor using green and violet laser are $0.78 \mathrm{mV} /(\mathrm{mg} / \mathrm{dL})$ and 0.72 $\mathrm{mV} /(\mathrm{mg} / \mathrm{dL})$, respectively. The highest accuracy is obtained for sensor with green laser. The accuracy of sensor with red, green, and violet laser are respectively $96.9 \%, 85.5 \%$, and $84.1 \%$.The accuracy of the developed sensor fulfills the requirement to be used by diabetic patients.

Keywords: urine, blood glucose sensor, fiber optic, evanescent wave, sensitivity, accuracy

\section{Introduction}

Diabetes mellitus is a condition where blood glucose level of a person is too high due to carbohydrate metabolism disorder occurring when body do not produce enough insulin or cannot used it effectively [1]. Diabetes is often found in medium to low income countries and urban societies because of unhealthy diet and lifestyle. Currently more 
to increase rapidly. Indonesia, with 12 million patients, is in the sixth place in term of diabetic patient number [2].

Regular blood sugar (glucose) level monitoring is vital for diabetic patients in order to keep glucose level within a normal range, to set medication plan, and to avoid complications. Glucose level measurement is mainly done by measuring electrical signals when blood sample reacts with a certain enzyme [3]. Blood sample collection by pricking patient finger is inconvenient and may lead to infection [4]. Infection may harm patients because their body do not produce insulin that is important for wound healing [5]. Therefore, non-invasive glucose level measurement using other body fluids is demanded. Among others, urine is more preferential because it can be collected easily and its sugar content correlates very well with that of blood [6].

Non-invasive measurements have been performed using many spectroscopic methods such as infra-red, Raman, and photo-acoustic [7, 8]. Infra-red spectroscopy faces difficulty in handling strong absorption of water molecules, Raman spectroscopy requires long measurement time, while photo-acoustic spectroscopy suffers from interference of bio-chemical signal. Fiber optic based sensor offers several advantages namely electromagnetic interference immunity, lightweight, compact, and high sensitivity [9].

Here we report the influence of diode laser wavelength on sensitivity and accuracy of evanescent wave based fiber optic sensor for blood glucose level measurement. The reasons are, first laser wavelength determines the penetration depth of evanescent wave and, second certain light spectrum can be absorbed by glucose molecules in urine [10]. Three diode lasers were used as a source for the sensor, they are red $(\lambda=$ $650 \mathrm{~nm})$, green $(\lambda=532 \mathrm{~nm})$, and violet $(\lambda=405 \mathrm{~nm})$.

\section{Materials and Methods}

\subsection{Material preparation}

The developed fiber optic sensors consist of a diode laser source, a multimode fiber optic, a photodiode, an arduino UNO microcontroller, and an LCD panel that are arranged as shown in Fig. 1. Light from diode laser is guided by the fiber optic to photodiode. Part of fiber optic cladding in the sensing area (urine sample) is removed to allow evanescent wave from fiber optic core to interact directly with glucose molecules in urine. The interaction causes attenuation of guided light in fiber optic core which is then detected by the photodiode. The attenuation is proportional to glucose level. The 
change in photodiode output was converted by arduino UNO into glucose level and then displayed on LCD panel.

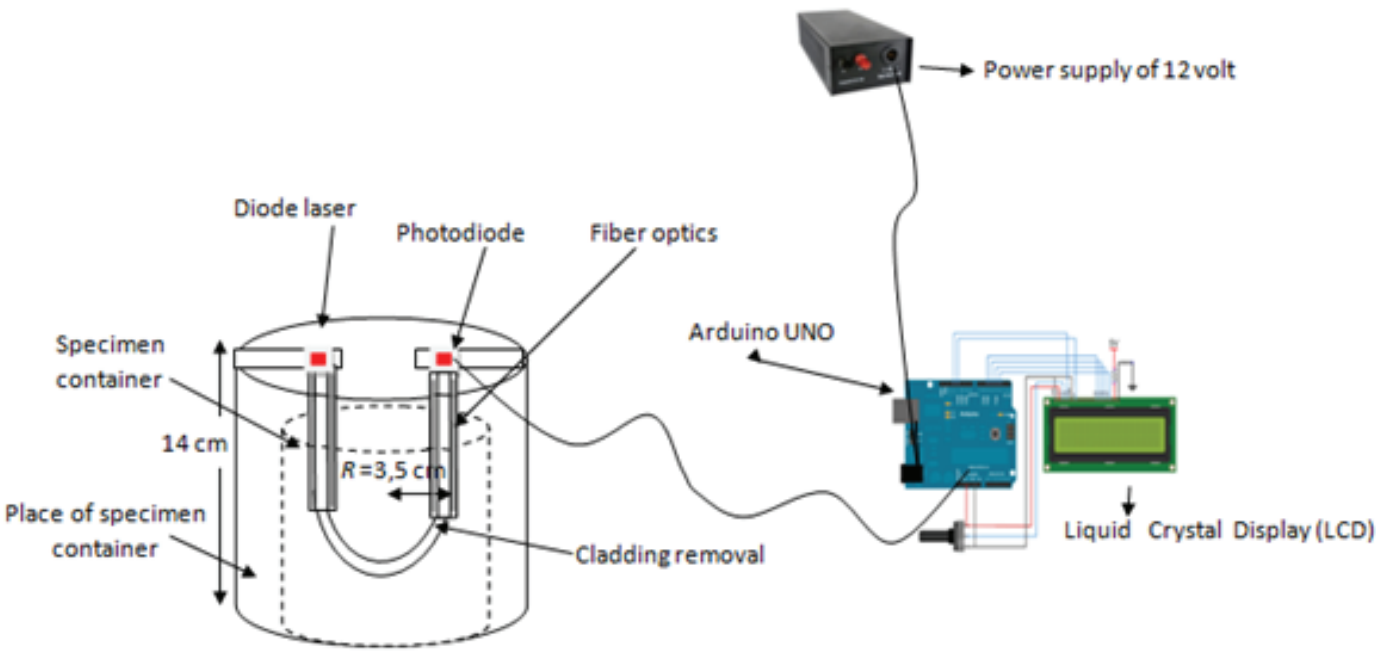

Figure 1: Measurement set up. Urine specimen is in contact with cladding-removed fiber optic.

The removal of fiber optic cladding was done first by removing jacket layer using a sharp cutter, then cladding is smoothly abraded using acetone-wetted tissue. The success in cladding removal is indicated by visible loss of guided light in sensing area. The loss was measured using a BF5R optical detector. The length of cladding removal was varied from $1 \mathrm{~cm}$ to $4 \mathrm{~cm}$.

Urine samples of diabetic patients were obtained from Dr. Reksodiwiryo Hospital, Padang. Before measurement, urine samples were reacted with benedict solution at 1:1 volume ratio. After mixing, urine samples were heated for $30 \mathrm{~s}$ until color change was observed. The color of urine after reaction indicates its glucose content.

\subsection{Characterization of fiber optic sensor}

To be used as a glucose level sensor, fiber optic sensor must first be characterized to obtain its transfer function. Transfer function represents correlation between photodiode output voltage and glucose concentration of reference urine samples. The transfer function was inserted into arduino UNO control program to convert measured photodiode voltage into glucose level. Transfer function is measured for all variation of laser diode wavelength and length of cladding removal. Slope of transfer function is no other than the sensitivity of fiber optic sensor. 


\subsection{Measurement of blood glucose}

Glucose level of urine sample is measured by using set-up shown in Fig. 1. Urine sample that was previously reacted with benedict solution was placed in specimen container and then un-cladded fiber optic was immersed into urine solution. Attenuated light by urine sample was detected by photodiode and then glucose level was displayed on LCD panel. After each measurement, sensing area of fiber optic was cleaned from remaining urine sample by spraying distilled water and then dried using soft tissue. The accuracy of fiber optic sensor was determined by measuring urine of diabetic patients and then compared it to the result of hospital standard test.

\section{Results and Discussion}

\subsection{Sensitivity of fiber optic sensor}

Fig. 2 displays the correlation between photodiode output voltage and reference glucose level for three different laser sources. This characterization is aimed at determining sensitivity of developed fiber optic sensor. Photodiode output voltage decreases linearly with glucose level in the range of $90-340 \mathrm{mg} / \mathrm{dL}$. Longer cladding removal results in lower output voltage. Photodiode voltage drops significantly with the wavelength of laser source.

Higher glucose concentration causes stronger scattering and absorption of evanescent wave by glucose molecules in urine so that guided light in fiber optic core decreases. The attenuation of guided light in fiber optic core results in a lowering in photodiode output voltage. Attenuation is also higher for short wavelength source. Short wavelength wasmore scattered and absorbed by glucose molecules.

The slope of transfer function indicates the sensitivity of fiber optic sensor. Fig. 3 shows that the highest sensitivity is obtained for sensor with red laser, followed successively by green and violet laser. Higher sensitivity of fiber optic with red diode laser is due to larger penetration depth of evanescent wave. Sensitivity of fiber optic sensor with red, green, and violet lasers are about $0.9 \mathrm{mV} /(\mathrm{mg} / \mathrm{dL}), 0.75 \mathrm{mV} /(\mathrm{mg} / \mathrm{dL})$, and $0.65 \mathrm{mV} /(\mathrm{mg} / \mathrm{dL})$, respectively. Sensitivity of fiber optic sensor varies slightly with the length of cladding removal. 

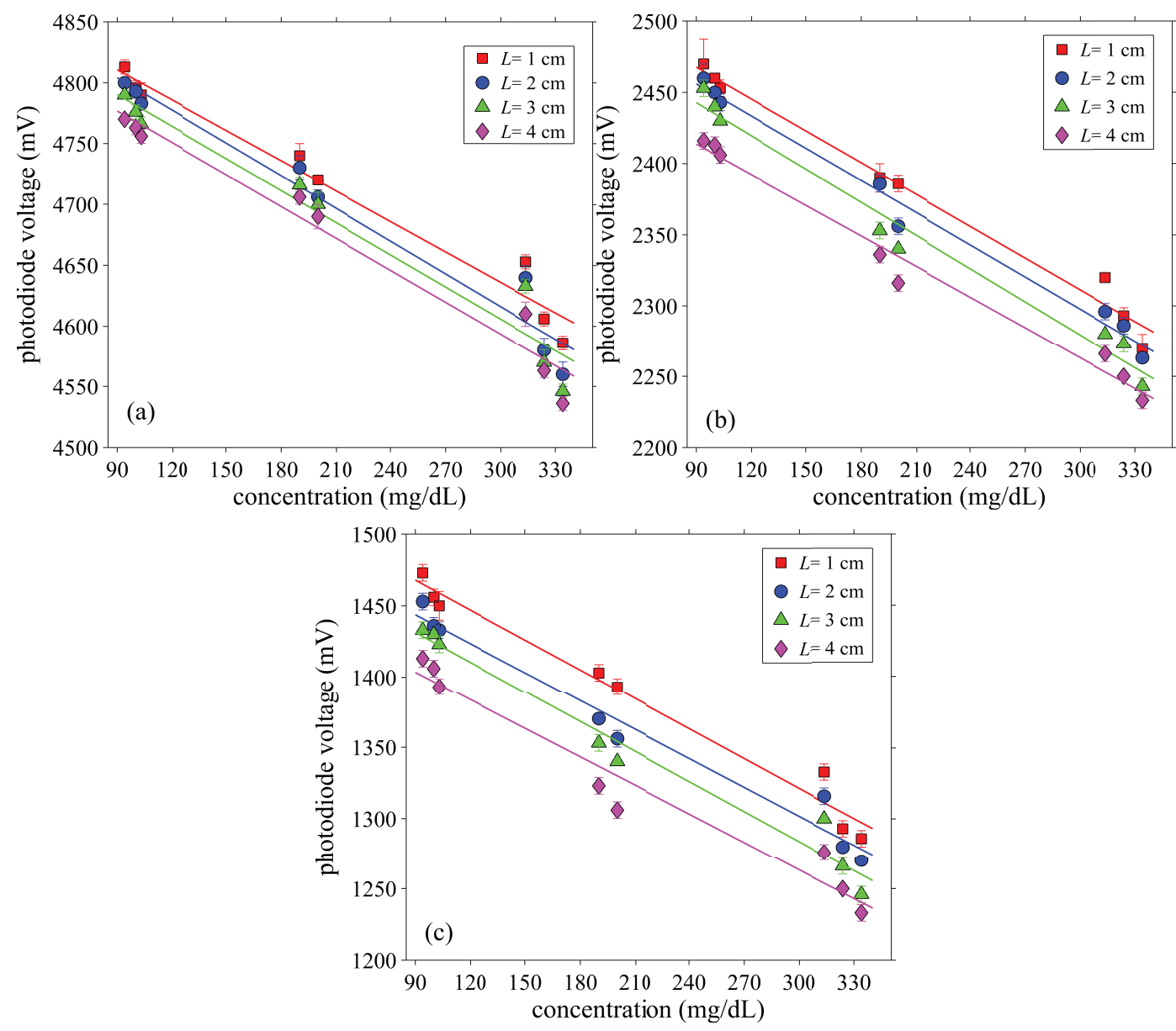

Figure 2: Dependency of photodiode output voltage on glucose concentration for different wavelength of diode laser, (a) red, (b) green, and (c) violet.

\subsection{Clarke error grid analysis}

Clarke Error Grid (CEG) is a standard way to present the accuracy of glucose sensor which relates measured and data from standard test. CEG plot is divided into several regions where region $A$ and $B$ indicate low measurement error, while on contrary $C$, $D$ and $E$ are regions of clinically intolerable measurement error [11]. Fig. 4 displays measured data from all variation of diode laser wavelength and length of cladding removal. All data for fiber optic sensor with violet and red lasers lie in region A (see Fig. 4.a and Fig. 4.c). Measured data from fiber optic sensor with green laser (Fig. 4.b) lie $97.5 \%$ in region $A$ and $2.5 \%$ in region $B$. In general we can say that the accuracy of developed fiber optic sensor fulfills the reliability requirement needed to be used by diabetic patients. 


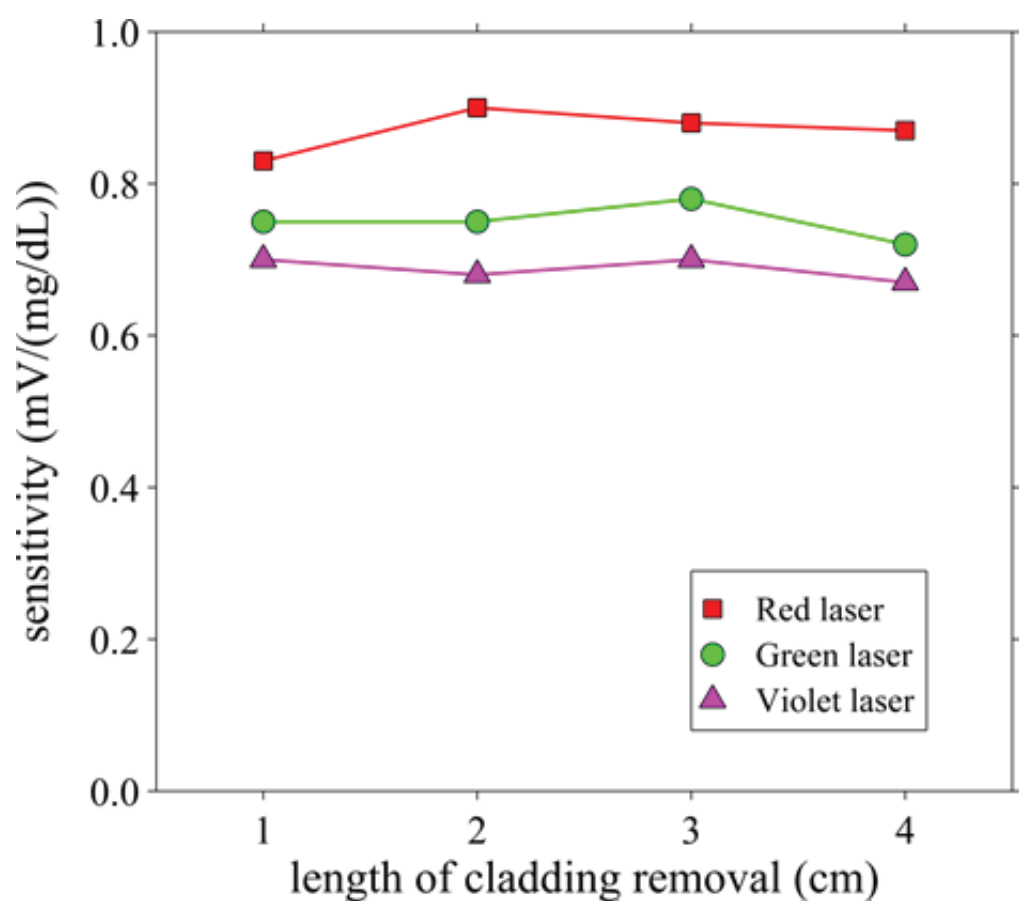

Figure 3: Sensitivity of fiber optic sensor as a function of the length of cladding removal for different laser wavelengths.

\subsection{Measurement accuracy}

Fig. 5 shows that measurement accuracy decreases slightly (within 1\%) with the length of cladding removal. Accuracy of fiber optic sensor with red laser is higher than that of green and violet laser. The highest accuracy of $96.9 \%$ is obtained for red laser with 4 $\mathrm{cm}$ cladding removal. A higher accuracy of fiber optic sensor with red laser source can be explained due to a lower light scattering and absorption.

\section{Conclusions}

We have shown that wavelength of diode laser affects sensitivity and accuracy of fiber optic sensor. Sensor using red diode laser shows higher sensitivity and higher accuracy due to a longer penetration depth of evanescent wave. Sensitivity and accuracy of fiber optic sensor increase slightly with the length of cladding removal. CEG analysis indicates that the developed sensor is reliable and accurate to be used by diabetic patients. 

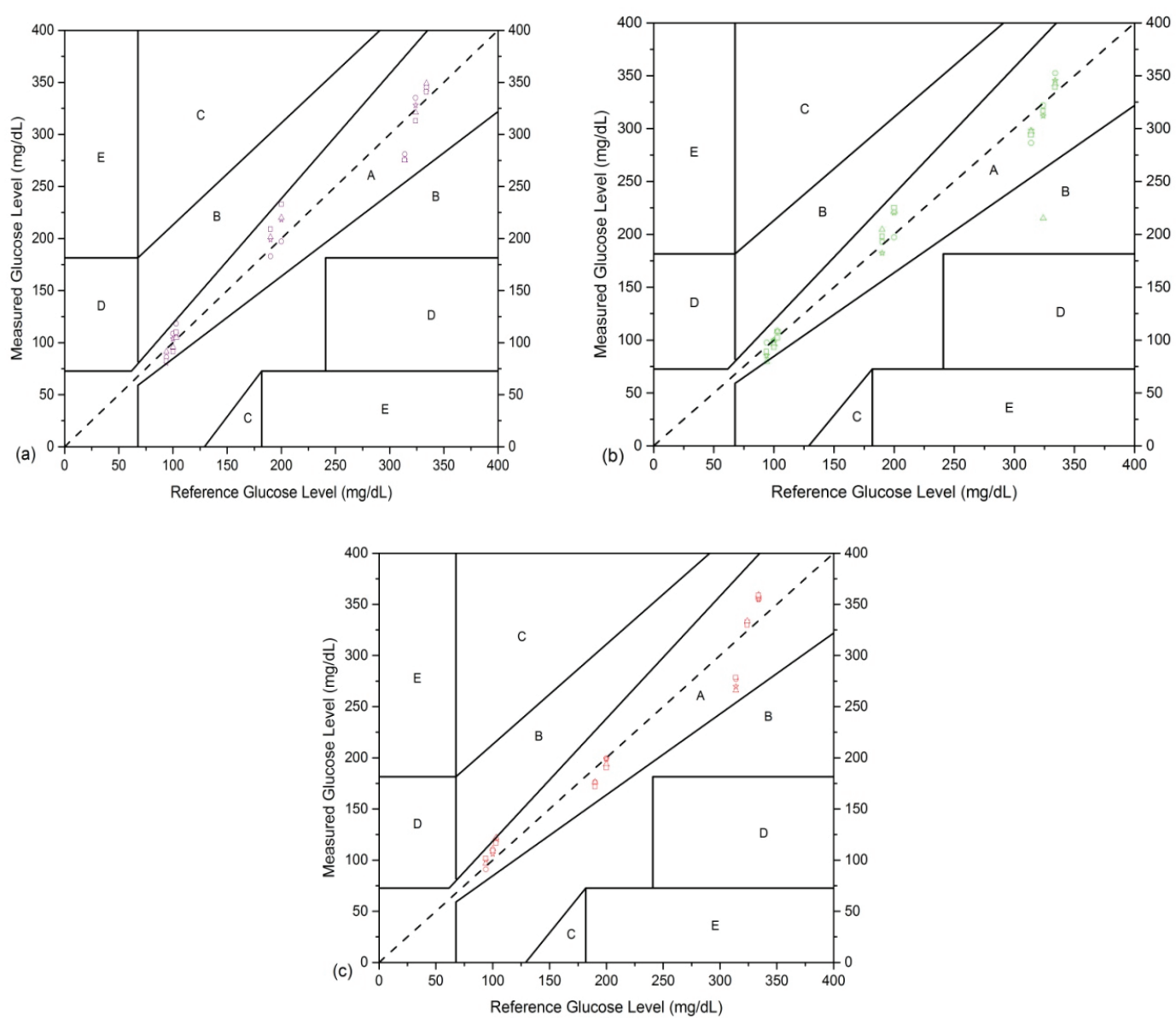

Figure 4: Glucose level measurement validation graph in form of Clark Error Grid for different wavelength of laser source, (a) violet, (b) green, and (c) red.

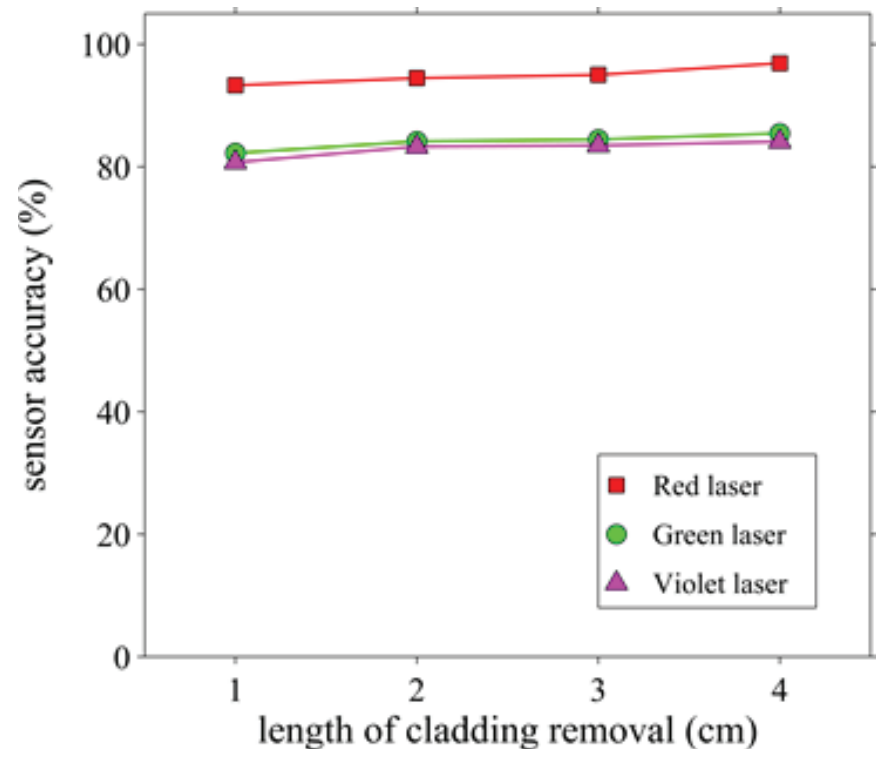

Figure 5: Accuracy of fiber optic sensor as a function of length of cladding removal for three different laser sources. 


\section{Acknowledgments}

This research was supported by the Grant from the Faculty of Mathematics and Natural Sciences of Andalas University with contract No.35/UN.16.03.D/PP/FMIPA/2017.

\section{References}

[1] Kerner, W., and Bruckel.,'Definition, Classification and Diagnosis of DiabetesMellitus",German diabetes Association: Clinical Practice Guidelines122, 384-386 (2014).

[2] International Diabetes Federation. IDF Diabetes At/as 8th ed. Brussel, Belgium (2017).

[3] Wang, H.-C. and Lee, A.-R., "Recent developments in blood glucose sensors“, Journal of food and drug analysis 23,191 - 200 (2015)

[4] Porte, D., "Central Regulation of Energy Homeostasis: The Key Role of Insulin", Diabetes, Vol.55. Supplement2, 155-160 (2006).

[5] Alan, R.S., and Kahn, R.,"Insulin Signalling and the Regulation of Glucose and Lipid Metabolism“,Nature414, 799-806 (2001).

[6] Sorvoja, H., andMyllyla, R., "Noninvasive Blood Pressure Measurement Methods", Optoelectronics and Measurement Techniques Laboratory27, 239-264 (2006).

[7] Boatemaa,M.A., and Srinath,D.,"Non-invasive Glucose Estimation Based on Near Infrared Laser Diode Spectroscopy“, Asian J. Biomed. Pharmaceut. Sci. 1 (60), 22-28 (2017).

[8] Yadav, J., Rani, A., Singh, V., and Murari, B. M., "Prospects and Limitations of Noninvasive Blood Glucose monitoring using Near-infrared Spectroscopy“, Biomedical Signal Processing and Control,18, 214 (2015).

[9] Mescia, L., and Prudenzano, F., "Advances on Optical Fiber Sensors",Fibers2, 1-23 (2014)

[10] Chowdhury, M.K., Anuj, S., Neeraj, S., and Shiru, S. "Error Grid Analysis of Reference and Predicted Blood Glucose Level Values as Obtained from The Normal and Prediabetic Human Volunteer“,American Journal of Biomedical Engineering5(1), 6-14 (2015).

[11] Pfutzner, A., David,C., Klonoff., Scott, P., and Joan, L.P.,“Technical Aspects of The Parkes Error Grid“,Journal of Diabetes Science and Technology7(5), 1275-1281 (2013). 